DOI $10.35381 / \mathrm{cm} . v 5 i 1.318$

\title{
Herramientas para el control de inventarios inteligentes en la industria del calzado de la provincia de Tungurahua
}

\section{Tools for the control of intelligent inventories in the footwear industry of the province of Tungurahua}

\author{
Milena Pazmiño Freire \\ pazmino13milena@gmail.com \\ Universidad Católica de Cuenca, Cuenca \\ Ecuador \\ https://orcid.org/0000-0002-8114-5011 \\ Cecilia Ivonne Narváez Zurita \\ inarvaez@ucacue.edu.ec \\ Universidad Católica de Cuenca, Cuenca \\ Ecuador \\ https://orcid.org/0000-0002-7437-9880 \\ Juan Carlos Erazo Álvarez \\ jcerazo@ucacue.edu.ec \\ Universidad Católica de Cuenca, Cuenca \\ Ecuador \\ https://orcid.org/0000-0001-6480-2270
}

Recibido: 07 de septiembre de 2019

Aprobado: 28 de septiembre de 2019

\section{RESUMEN}

El manejo de inventarios constituye el eslabón más importante de las empresas, sin lugar a dudas los avances tecnológicos y la sistematización de los procesos han contribuido en la última década a su modernización. El disponer de un inventario preciso tiene un fuerte impacto sobre los resultados económicos de las empresas y la satisfacción de los clientes más aún si se busca la diferenciación en la industria nacional de calzado. Por consiguiente el objetivo de este artículo es diagnosticar la gestión y control de inventarios de las pequeñas y medianas empresas productoras de calzado, localizadas en el Catón Cevallos, provincia de Tungurahua. Para ello, la investigación se sustentó en un estudio no experimental, con corte transeccional y alcance descriptivo - explicativo. Entre los principales resultados se determinó la inexistencia de softwares especializados para el 
control de inventarios, la no ejecución de controle físicos permanentes, la deficiente ubicación del inventario en los almacenes, la falta de información en tiempo real para la toma de decisiones y el aprovisionamiento en base a la intuición y experiencia.

Descriptores: gestión; control; inventarios, valuación, inteligencia artificial.

\begin{abstract}
Inventory management is the most important link in companies, without a doubt the technological advances and systematization of the processes have contributed in the last decade to its modernization. Having an accurate inventory has a strong impact on the economic results of companies and customer satisfaction even more if differentiation is sought in the national footwear industry. Therefore, the objective of this article is to diagnose the management and control of inventories of small and medium-sized companies that produce footwear, located in Caton Cevallos, province of Tungurahua. For this, the research was based on a non-experimental study, with a transectional cut and descriptive - explanatory scope. Among the main results was the absence of specialized software for inventory control, the non-execution of permanent physical controls, the poor location of inventory in warehouses, the lack of real-time information for decision making and provisioning in based on intuition and experience.
\end{abstract}

Descriptors: management; control; inventories, valuation, artificial intelligence.

\title{
INTRODUCCIÓN
}

El control de inventarios se ha constituido en uno de los elementos más complejos de la logística y de la administración de la cadena de abastecimiento, siendo una de las dificultades típicas la existencia de excesos y los faltantes de inventarios, esta situación es un problema latente en empresas industriales, comerciales o de servicios, las mismas que disponen y administran insumos, materias primas, repuestos, productos en proceso y productos terminados, lo que les obliga a mantener cantidades de inventario en mayores o menores niveles. Al respecto, las causas que originan la necesidad de mantener inventarios, son las fluctuaciones aleatorias de la demanda por parte de los consumidores y los tiempos de reposición por parte de los proveedores (Vidal, 2010), no obstante, considerado que las causas que obligan a mantener inventarios no pueden ser 
eliminadas totalmente, la mejor opción es establecer sistemas óptimos de gestión y control, en este sentido, la gestión se puede ayudar de una serie de técnicas cualitativas o inteligentes que facilitan dicho control, Arraiz (2018).

La mayoría de las técnicas cuantitativas se sustentan en modelos matemáticos que contribuyen a la toma de decisiones en inventarios, mientras que, las técnicas inteligentes se respaldan en plataformas que integran y automatizan distintas prácticas de negocio relacionadas con aspectos productivos, operativos y de logística, Chirinos (2016).

Lo anteriormente expuesto, de la mano con la apertura de mercados que actualmente se vive, obliga a que las empresas locales del Ecuador avancen al ritmo que exige el mercado internacional, para evitar su rezago frente a la voraz competencia, en tal virtud, hoy, mantener inventarios en línea y automatizados, es una estrategia esencial para alcanzar una alta participación en el mercado. Frente a esta realidad, el presente artículo tiene por objetivo analizar la sintomatología de la ausencia de controles de inventarios de las pequeñas y medianas empresas dedicadas a la producción y comercialización de calzado en la provincia de Tungurahua.

\section{DESARROLLO}

Toda empresa que desea alcanzar el éxito en el mercado, debe lograr una conjugación razonable de calidad, oportunidad, costos y cantidad de los bienes y servicios que produce. Cuando se habla de oportunidad en el aprovisionamiento y la entrega, definitivamente la gestión y el control de los inventarios, juegan un rol fundamental ya sea en el caso de materias primas, productos en proceso o productos terminados. Para ello se requiere aplicar criterios de planificación a fin de obtener resultados eficientes.

En esta investigación se precisara la gestión de inventarios en los almacenes, los mismos que tienen como características significativas la venta de productos finales y el desconocimiento de la demanda futura.

Bajo este escenario, Martínez (2006) afirma que los almacenes que comercializan productos terminados se enfrentan con dificultades relacionadas al conocimiento de 
cuándo y cuánto comprarán los clientes, a pesar de ello, el autor resalta la importancia de establecer patrones de comprotamiento, para determinar el grado de probabilidad de ventas futuras. Bajo estas condiciones, la gestión de inventarios consiste en establecer la cantidad y el tiempo en que se deben reponer los productos en un almacén, considerando que un error en cantidades exesivas puede producir sobrealmacenamiento e incremento de los costos, mientras que, si la cantidad de respuesta es baja puede acarrear desabastecimiento y como consecuencia pérdida de clientes.

\section{Gestión de inventarios}

Los inventarios corresponden a inversiones que realizan las empresas y representan un porcentaje significativo en el activo circulante, por lo que su control y manejo debe ser realizado de forma minuciosa, a efectos de maximizar su preservación y custodia, al respecto, Sierra, Guzmán, y García, 2017, explican que un ineficiente manejo de inventarios, trae como consecuencia excesos, desperdicios y variabilidad. En el caso de los excesos estos se presentan cuando existe un elevado nivel de inventarios que está en espera de ser vendido en un lapso mayor a uno o dos meses; en cuanto a los desperdicios, los mismo se exteriorizan cuando hay probabilidad de que los materiales se deterioren o caduquen; y, la variabilidad es un inconveniente que se da por la prolongación del horizonte de la planeación, lo que genera mayor incertidumbre, que en ocasiones conlleva a disponer de productos que no se necesitan.

En términos generales se puede considerar al inventario como la relación detallada, clasificada y valorada de cada uno de los elementos que se encuentran en el punto de venta en un momento determinado, por consiguiente, es un elemento imprescindible en la contabilidad de todo tipo de organización. El inventario tiene por finalidad evitar que la falta de productos deje de satisfacer la demanda, además de generar oportunidades de mejora en la gestión de inventarios; junto a estos que son los principales objetivos del inventario, resulta interesante reconocer otros objetivos, considerando el análisis de 
varios autores (Guerra, 2010; Peña y Silva, 2016; Cruz, 2017; Juca, Narváez, \& Erazo, 2019) :

- Reducir costos de distribución.

- Abaratar las compras a través de pedidos por lotes.

- Eliminar productos caducados, deteriorados o rotos.

- Disminuir las variaciones entre la oferta y la demanda.

- Reducir los riesgos manteniendo stocks ${ }^{1}$ de seguridad.

Lo antes expuesto, deja en evidencia que la gestión de inventarios constituye uno de los eslabones más importantes de las organizaciones ya que de ello depende el adecuado abastecimiento de los clientes, por ende, se deben tener consideraciones importantes relacionadas con su direccionamiento estratégico, la visión administrativa y el entorno, para así generar una función lógica del manejo de los inventarios y disponer de un equilibrio entre el suministro y la demanda, en consecuencia, es indispensable establecer los lineamientos o filosofía bajo la cual se determinará la cantidad a ordenar y el momento que se hará la orden (Zapata, 2014) .

Ahora bien, para definir estos lineamientos se debe estimar previamente los costos de inventarios, que hacen referencia a todos los gastos asociados a disponer de stocks en la bodega, la clasificación del stock cuyo fin es diferenciar los bienes que forman parte del inventario de acuerdo a criterios establecidos y finalmente la asignación de inventario de seguridad.

La gestión de inventarios debe establecer el equilibrio entre la cantidad y los costos derivados de mantener inventarios. Para lograr este fin, en la tabla 1 se presentan los distintos costos de los inventarios y sus componentes.

\footnotetext{
1 Stock: bienes o productos que la empresa necesita mantener almacenados para su posterior venta
} 
Tabla 1. Costos de inventario

\begin{tabular}{ll} 
Costo & Componentes del costo \\
\hline Capital & $\begin{array}{l}\text { Corresponde a la pérdida de valor de los inventarios con } \\
\text { relación al tiempo. }\end{array}$ \\
\hline Impuestos & Son los gastos en impuestos que se paga por adquirir y \\
& mantener los inventarios. \\
\hline Seguros & Son los valores que debe pagar la empresa por deterioro, \\
& accidentes, pérdidas y otros. \\
\hline Obsolescencia & Se da por la pérdida de la mercadería cuando su tiempo \\
& de vida ha caducado. \\
\hline Almacenamiento & Los costos de almacenamiento incluyen: costos de \\
& espacio, mano de obra , energía, costos de infraestructura
\end{tabular}

Fuente: (Zapata, 2014)

En la literatura especializada se identifican varias técnicas para administrar el inventario, cuyo propósito principal es disminuir los costos y maximizar las utilidades. Al respecto, Bustos y Chacón (2007) señalan que las empresas deben seleccionar las técnicas para administrar los inventarios de acuerdo a la naturaleza de la demanda, es decir, ya sea esta dependiente o independiente. La demanda dependiente se genera a partir de las necesidades derivadas directamente de los requisitos de otros bienes inventariados 0 producidos en la empresa, mientras que, la demanda independiente está sujeta a las condiciones de mercado

En todo tipo de organización es necesario realizar una discriminación de artículos, a fin de establecer aquellos que por sus características demandan de controles más rigurosos, en este contexto, la clasificación $A B C$ constituye una metodología que segmenta los productos de acuerdo a criterios preestablecidos tal como se aprecia en la tabla 2. 
Tabla 2. Clasificación ABC

\begin{tabular}{l|lll}
\multicolumn{1}{c}{ A } & \multicolumn{2}{c}{ B } \\
\hline Productos de alto valor, con & Productos de alto valor, con & Productos de bajo valor, \\
gran volumen de ventas, que & ventas moderadas, necesita & pocas ventas, se deben tratar \\
requieren mayor cuidado. & de un tratamiento normal, bajo el principio de \\
Para ello se debe: & esto quiere decir, que la & simplificación productiva y \\
-Analizar en el mercado los & atención debe estar ajustada & administrativa, y reducción de \\
precios y costos. & a los requerimientos de la & costos. \\
-Registrar y controlar los & organización. & -Simplificación de inventarios. \\
inventarios. & & -Simplificación en el manejo \\
-Determinar de forma precisa & & de pedidos. \\
\hline las exigencias de seguridad. & & -Simplificación en la \\
-Aplicar el análisis de valores. & & supervisión de existencias. \\
\hline
\end{tabular}

En varias investigaciones ( (Parada, 2009; Castro, Vélez, y Castro, 2011; Toro y Bastidas, 2011; Rodríguez y Gutiérrez, 2016; Veloz y Parada, 2017; Cardona, Orejuela, y Rojas, 2018) se ha establecido que el nivel de importancia de un producto debe ser considerado tomando en cuenta las ventas anuales, el costo unitario, la oferta y la demanda, las condiciones de almacenamiento, el riesgo de obsolescencia o caducidad, la confiabilidad de los proveedores, tiempo de entrega , reparabilidad, entre otros. Se puede apreciar al respecto, que no existe un consenso entre los autores para definir los criterios a considerar en la clasificación $A B C$, por lo que en este punto se reafirma la importancia del buen juicio de la dirección.

De modo general, en la literatura especializada la metodología $A B C$ se apoya en métodos como el análisis jerárquico, la optimización lineal, análisis matricial, arboles de decisión y reglas heurísticas, de esta manera la clasificación $A B C$ presenta ciertas restricciones relacionadas con su alto nivel de complejidad para tomar decisiones a nivel operativo, debido a la gran cantidad de datos a considerar. 


\section{Control de inventarios}

Arbós (2012) señala que el control de inventarios consiste en manejar adecuadamente los registros, la rotación y la evaluación del inventario de acuerdo a la clasificación que se realice y al tipo de inventario con el que cuenta la empresa, cuya finalidad principal consiste en determinar la cantidad de inventario que debe mantenerse, la fecha en que deben colocarse los pedidos y las cantidades a ordenar. De conformidad con lo expuesto, Velázquez (2012) menciona que los controles sobre el inventario inician cuando se reciben los productos, de tal manera que, el departamento responsable de la recepción presentará los informes correspondientes para proceder con la contabilización del inventario, esto permitirá verificar que los productos que llegaron a la empresa son los que realmente se solicitó al proveedor

En lo referente al registro de los inventarios, contablemente existen dos sistemas, el periódico y el perpetuo.

El inventario periódico se aplica por lo general a las empresas que comercializan artículos relativamente económicos, por lo que levantan periódicamente su inventario, al menos una vez al año con el fin de establecer la cantidad en existencias y elaborar los estados financieros. En este sistema el costo de ventas se determina como el resultado de la siguiente ecuación:

Costo de ventas $=$ Inventario inicial + Compras y costos - Inventario final El inventario perpetuo se aplica en empresas que comercializan productos de alto costo y que requieren mantener un registro al día sobre sus existencias, por consiguiente la empresa debe actualizar el importe de sus inventarios cada vez que realice operaciones de compra o venta. Con la aplicación de este sistema, el inventario final que consta en los registros contables debe ser comprado con el conteo físico en el periodo que la entidad estime, lo cual permite mantener un mayor control sobre estos activos 
Las Normas Internacionales de Información Financiera, por su parte, en la sección 13 Inventarios establecen los principios para el reconocimiento y medición de los inventarios, para ello identifican con tal a los bienes mantenidos para la venta en el curso normal de las operaciones, a los bienes en proceso de producción con destino a ser vendidos, y, a los materiales o suministros a ser consumidos durante el proceso de producción. Los métodos de valoración y fórmulas de costeo que reconocen estas normas son: a) identificación específica de sus costos individuales (Retail); b) Método promedio ponderado; y, c) Método FIFO o PEPS (IASB, 2015).

Weygandt, Kimmel y Kieso (2009) mencionan que una empresa que utilice el método de identificación específica deberá mantener en sus registros contables los costos originales de cada objeto, por su parte, Carmichael, Whittington y Graham (2007) señalan que una entidad que utilice la fórmula de primeras entradas, primeras salidas (PEPS), tendrá que asumir que sus inventarios ingresaran y saldrán cronológicamente de tal forma que se prevendrá la obsolescencia de los productos. La IASB (2015) indica que las empresas que utilicen el método de promedio ponderado deberán determinar el costo de cada unidad de producto a partir del promedio ponderado del costo de los artículos similares poseídos al inicio del período y del costo de los mismos artículos comprados o producidos durante el período.

Como se puede apreciar cada una de las formulas establece un tratamiento distinto para la valuación de los inventarios, por lo que la aplicación de cualquiera de ellas producirá impactos distintos en los estados financieros y en el importe reconocido como impuesto a la renta.

\section{La inteligencia artificial en la optimización de inventarios}

Actualmente a nivel mundial los procesos de gestión de inventarios se están modernizando a causa de los avances tecnológicos y de la sistematización, es así que se han desarrollado técnicas de inteligencia artificial que contribuyen a mejorar las 
estrategias de las organizaciones en relación a la precisión, control y ejecución de inventarios en tiempo real, a través de imágenes, códigos y videos, que permiten identificar de forma automática la cantidad y ubicación de las existencias (Schwarz, 2018)

Al respecto, los sistemas informáticos modernos buscan controlar con eficiencia los inventarios con la finalidad de mantener existencias mínimas y mejorar las finanzas de la organización, no siendo estos los únicos beneficios que otorgan las nuevas tecnologías ya que se puede lograr ahorro en inventarios invirtiendo únicamente en lo necesario para disminuir costos y espacio de almacenamiento; se consigue incrementar la productividad evitando la interrupción de operaciones o la pérdida de ventas por desabasto; es posible reforzar la seguridad y prevenir fugas de inventario, mediante sistemas avanzados de monitoreo; y, finalmente se puede mejorar la información a través de la generación de reportes personalizables capaces de identificar tendencias de consumo.

Al respecto, según Velázquez (2012) existen herramientas tecnológicas que pueden contribuir con el cumplimiento de las tareas expuestas en el párrafo que antecede, como por ejemplo:

Etiquetas con tecnología dual: consisten en pequeños dispositivos fáciles de usar que se adhieren a los productos, las mismas transmiten la identidad del objeto mediante ondas de radio frecuencia (RFID).

Sistemas de administración de almacén: permiten automatizar procesos para optimizar el inventario y preparar pedidos.

Softwares: existen softwares que permiten controlar devoluciones, crear facturas, codificar productos, manejar bases de datos, generar órdenes de compras automáticas, rastrear las ventas, visualizar la existencia de los inventarios, entre otras opciones.

Almacenes 4.0: se refiere a soluciones automatizadas flexibles e inteligentes que permiten una interconexión entre la robótica y la gestión de interacción con las personas. 
Aplicaciones ERP: la planificación de recursos empresariales consiste en una aplicación que integra sistemas de gestión y se utilizar para controlar el inventario, además este sistema permite facturar los pedidos.

Lectura de códigos: es un sistema de lectura capaz de leer letras, números o combinaciones de los dos.

Para llevar a cabo una adecuada implementación de inventarios inteligentes se debe considerar las siguientes estrategias:

I. Estandarizar los procesos a través de un catálogo normalizado.

II. Registrar los parámetros de catálogo e incluir imágenes de los productos.

III. Digitalizar las imágenes.

IV. Desarrollar procesos de entrenamiento sobre el catálogo normalizado.

V. Establecer fechas de corte.

VI. Realizar pruebas de operación.

VII. Obtener reportes predictivos.

VIII. Sincronizar los stocks entre almacenes.

\section{Indicadores de gestión para el proceso de gestión de inventarios}

Contar con herramientas para evaluar el desempeño del inventario es fundamental para la administración del stock. Al respecto, Zapata (2014) afirma que la gestión por indicadores es una herramienta práctica que permite medir la relación de distintas variables para determinar el desempeño y comportamiento de los productos a almacenar. A continuación, en la tabla 3 se presenta un guía de indicadores financieros, operativos y de servicio al cliente para la administración y control del inventario.

Tabla 3. Indicadores de gestión

\begin{tabular}{|l|c|l|}
\hline \multicolumn{1}{|c|}{ Indicador } & \multicolumn{1}{|c|}{ Ecuación } & \multicolumn{1}{c|}{ Interpretación } \\
\hline $\begin{array}{l}\text { Porcentaje de } \\
\text { activo }\end{array}$ & Porcentaje de Activos $=\frac{\text { Valor inventario Físico }}{\text { Activos fijos }} \times 100 \%$ & $\begin{array}{l}\text { Representa el porcentaje } \\
\text { de activos que } \\
\text { corresponde al inventario } \\
\text { (Porcentaje). }\end{array}$ \\
\hline
\end{tabular}


CIENCIAMATRIA

Revista Interdisciplinaria de Humanidades, Educación, Ciencia y Tecnología

Año V. Vol. V. №1. Edición Especial. 2019

Hecho el depósito de ley: pp201602FA4721

ISSN-L: 2542-3029; ISSN: 2610-802X

Universidad Nacional Experimental Francisco de Miranda (UNEFM). Santa Ana de Coro. Venezuela

Milena Pazmiño Freire; Cecilia Ivonne Narváez Zurita; Juan Carlos Erazo Álvarez

\begin{tabular}{|c|c|c|}
\hline $\begin{array}{l}\text { Valor económico } \\
\text { del inventario }\end{array}$ & Valor económico del inventario $=\frac{\text { Valor inventario Físico }}{\text { Valor costo de ventas en el mes }}$ & $\begin{array}{l}\text { Identifica el valor del } \\
\text { inventario en relación a } \\
\text { las ventas (Unidad } \\
\text { monetaria). }\end{array}$ \\
\hline $\begin{array}{l}\text { Porcentaje de } \\
\text { ventas perdidas }\end{array}$ & Porcentaje de ventas perdidas $=\frac{\text { Valor ventas perdidas por falta de inventario }}{\text { Ventas Totales }}$ & $\begin{array}{l}\text { Detecta el efecto de no } \\
\text { disponer de un adecuado } \\
\text { abastecimiento para } \\
\text { hacer frente a la } \\
\text { demanda (Porcentaje). }\end{array}$ \\
\hline $\begin{array}{l}\text { Rotación del } \\
\text { inventario }\end{array}$ & Rotación de inventario $=\frac{\text { Ventas acumuladas }}{\text { Inventario promedio }}=$ número de veces & $\begin{array}{l}\text { Mide las veces en que la } \\
\text { mercadería rota en una } \\
\text { empresa (Número de } \\
\text { veces). }\end{array}$ \\
\hline $\begin{array}{l}\text { Porcentaje de } \\
\text { inventario sin } \\
\text { rotación }\end{array}$ & Porcentaje inventario sin rotación $=\frac{\text { Valor inventario sin movimiento último ańo }}{\text { Valor inventario promedio último ańo }} \times 100 \%$ & $\begin{array}{l}\text { Refleja la cantidad de } \\
\text { mercadería que no está } \\
\text { rotando en la bodega en } \\
\text { un año (Porcentaje). }\end{array}$ \\
\hline $\begin{array}{l}\text { Duración del } \\
\text { inventario }\end{array}$ & Duración del inventario $=\frac{\text { Inventario final }}{\text { Ventas promedio }} \times 30$ días & $\begin{array}{l}\text { Determina el tiempo que } \\
\text { la mercadería se } \\
\text { mantiene en inventario } \\
\text { (tiempo) }\end{array}$ \\
\hline $\begin{array}{l}\text { Inventario no } \\
\text { disponible }\end{array}$ & Inventario no disponible $=\frac{\text { Unidades dańadas }+ \text { Obsoletas }+ \text { Vencidas }}{\text { Unidades disponibles en inventario }}$ & $\begin{array}{l}\text { Determina el porcentaje } \\
\text { de mercaderías que no } \\
\text { están disponibles para la } \\
\text { venta a causa de daño, } \\
\text { obsolescencia o } \\
\text { vencimiento (Porcentaje). }\end{array}$ \\
\hline Nivel de servicio & $\begin{aligned} \text { Nivel de servicio por Unidad } & =\frac{\text { Unidades despachadas }}{\text { Unidades perdidas }} \\
\text { Nivel de servicio por referencia } & =\frac{\text { Referencias despachadas }}{\text { Total de Referencias perdidas }} \\
\text { Nivel de servicio por Ordenes } & =\frac{\text { Órdenes despachadas }}{\text { Total de órdenes perdidas }}\end{aligned}$ & $\begin{array}{l}\text { Mide el cumplimiento de } \\
\text { los requerimientos } \\
\text { realizados por los } \\
\text { clientes (Unidades } \\
\text { Referencia } \\
\text { Órdenes). }\end{array}$ \\
\hline $\begin{array}{l}\text { Tasa de } \\
\text { abastecimiento } \\
\text { de pedidos }\end{array}$ & Exactitud de abastecimicnto por pedido $=\frac{\text { Pedidos abastecidos correctamente }}{\text { Total de Pedidos Abastecidos }} \times 100 \%$ & $\begin{array}{l}\text { Analiza las ordenes que } \\
\text { han sido abastecidas a } \\
\text { los clientes sin } \\
\text { inconvenientes } \\
\text { (Porcentaje). }\end{array}$ \\
\hline
\end{tabular}

Fuente: Adaptado de Zapata (2014)

\section{METODOLOGÍA}

La presente investigación se enmarco en un enfoque mixto, ya que se combinaron los métodos cualitativos y cuantitativos para un mejor tratamiento de la información. En cuanto al alcance el estudio fue descriptivo explicativo ya que en una primera etapa se 
analizó la literatura para comprender la gestión de inventarios, sus modelos de valuación y las técnicas innovadoras de control. El diseño se fundamentó en un estudio no experimental, con corte transeccional, mediante el levantamiento de la información en un solo momento del tiempo y sin ningún proceso de manipulación.

Se consideró como unidades de análisis a las pequeñas y medianas empresas productoras y comercializadoras de calzado del Cantón Cevallos, provincia de Tungurahua.

Como instrumentos de recolección de información se aplicó la encuesta y la entrevista, la encuesta se realizó a los responsables del manejo de los inventarios y la entrevista a los administradores - propietarios, quienes accedieron voluntariamente a participar en la investigación.

El cuestionario estuvo estructurado en base a 10 preguntas con respuestas de selección múltiple previamente establecidas, el mismo fue aplicado a través de una prueba piloto a 12 propietarios de las PYMES productoras y comercializadoras de calzado, quienes fueron seleccionados por su reconocimiento y prestigio en el mercado. Como resultado de este proceso se obtuvo un $90 \%$ de aceptación de las preguntas y un $10 \%$ de observaciones. Una vez reestructurado el cuestionario se procedió con la aplicación del mismo a los 42 locales ubicados en el Cantón Cevallos.

\section{RESULTADOS}

Entre los principales resultados que arrojaron los instrumentos de investigación están los siguientes:

Control regular: El $43 \%$ de los locales comerciales cuentan con un software especializado que permite identificar la cantidad de pares de calzado existentes, sin embargo, sólo el $21 \%$ aseguran que los datos expuestos por el software coinciden con su inventario físico, ya que realizan controles y comparaciones al menos trimestralmente. El $37 \%$ afirman disponer de un sistema de entradas y salidas de productos, gestionado 
por el área contable mediante la aplicación de la herramienta Excel. Tan sólo el 20\% señalan no contar con ningún tipo de control regular sobre sus inventarios.

Inventario Físico: En el 87\% de locales comerciales se efectúa un inventario físico completo al menos una vez al año, con la finalidad de comprobar la existencia en inventarios y comparar con los registros contables. Dicho proceso lo realizan de forma programada al menos con diez días previos al término del año, ya que 29, 30 y 31 de diciembre son días de alta demanda en los que se realizan únicamente actividades relacionadas con las ventas.

Control ABC: Sólo el 15\% de encuestados afirman que utilizan el análisis ABC como herramienta para gestionar el inventario, por ende, realizan una clasificación de los productos que requieren mayor atención, ubicando en la categoría $A$ los productos de alto precio y baja frecuencia de ventas; en la categoría $B$ los productos de precio moderado, con frecuencia de ventas moderada; $y$, en la categoría $C$ los productos de precio bajo con alto volumen de ventas. Este resultado permitió determinar el bajo nivel de conocimiento con relación a este modelo por parte de los trabajadores y administradores - propietarios.

Dificultades en el control de inventarios: Al menos el $84 \%$ de los encuestados coinciden en que entre los principales problemas que enfrentan en la gestión de sus inventarios están: la deficiente organización de los productos en el almacén y su clasificación de acuerdo al nivel de rotación, la falta de registro oportuno de las transacciones que implican entradas o salidas del inventario, la carencia de tecnologías que permitan simplificar las tareas de control de inventarios, el exceso de inventario de diseños que ya no están en moda, el robos y el desorden en las bodegas.

Análisis de la demanda: El $68 \%$ afirman tener problemas para determinar la demanda de los modelos de calzado no relacionados con la estacionalidad escolar, por lo que, tienen dificultades para establecer la cantidad óptima a mantener en sus almacenes para atender la posible demanda sin generar excesos inútiles. 
Sistema de control de existencias: el $94 \%$ de los locales comerciales no mantiene ningún nivel de automatización para controlar su stock, por lo que no existe una codificación estandarizada previa, bajo código de barras, código de barras bidimensional o etiquetas electrónicas por radiofrecuencia. Al respecto se deduce, que en la mayoría de almacenes se manejan sistemas de control tradicionales.

Valoración de existencias: El 20\% de los locales comerciales aplica el método promedio ponderado para valorar sus inventarios; el 21\% utiliza el método FIFO (primeras en entrar , primeras en salir) ya que consideran que el calzado es un producto que responde a la moda; el 38\% utilizan el método LIFO (ultimas en entrar, primeras en salir), ya que piensan que el calzado es un producto que no se caduca o perece, mientras que, el $21 \%$ restante señala que no realizan la valoración de sus inventarios bajo ninguna técnica contable, debido a que por el monto de su capital no están obligados a llevar contabilidad. Ubicación del inventario: El 84\% de los locales comerciales no cuentan con el espacio físico suficiente que permita mantener un tratamiento específico y adecuado a sus necesidades, por lo que en varias ocasiones deben enfrentar mermas de calidad por el excesivo acopio del stock.

Gestión de la información: El $40 \%$ de encuestados mencionan que no dispone de información certera en tiempo real lo cual trasciende en un aprovisionamiento por intuición o por experiencia, sin ningún tipo de soporte técnico - numérico lo que conduce a que mantenga un stock excesivo en ciertos modelos de calzado y por defecto un bajo stock en otros.

Ventajas de mantener un control de gestión de inventarios: El 90\% afirman que mantener un control de inventario adecuado les generará beneficios en: reducción de inventario, disminución de costos de almacenaje, aprovechamiento de espacios, precisión en la disponibilidad de inventario, mejora de los procesos de control.

Los resultados expuestos dejan en evidencia la necesidad de reducir los costos de las operaciones diarias por parte de las PYMES productoras y comercializadoras de calzado del Cantón Cevallos, provincia de Tungurahua y así incrementar los beneficios para los 
propietarios, constituyendo a las actividades de gestión y control de inventarios en el foco de atención para alcanzar estos objetivos. Por consiguiente, se propone a continuación un esquema de gestión y control para los productos depositados en bodega y en los puntos de venta (almacenes comerciales) con la finalidad de reducir la cantidad de inventario requerido y elevar los niveles de cumplimiento con los clientes minoristas y mayoristas (Ver figura 1).

Además, considerando que una gestión y control de inventarios adecuada requiere de una participación armónica y activa de distintas áreas de las PYMES (compra, bodega, ventas, contabilidad, auditoría, finanzas) se propone como objetivos de este esquema: disminuir los costos operativos, reducir los costos por inventarios y mejorar el servicio al cliente, mediante la participación activa y armónica de las áreas de: bodega, contabilidad, finanzas, ventas y auditoría.

Finalmente el esquema propuesto se diseñó bajo una función lógica de la gestión y control de inventario de tal manera que las PYMES mantengan un equilibrio entre el suministro y la demanda, que permita atender permanentemente a los clientes con un excelente nivel de servicios. 
CIENCIAMATRIA

Revista Interdisciplinaria de Humanidades, Educación, Ciencia y Tecnología

Año V. Vol. V. №1. Edición Especial. 2019

Hecho el depósito de ley: pp201602FA4721

ISSN-L: 2542-3029; ISSN: 2610-802X

Universidad Nacional Experimental Francisco de Miranda (UNEFM). Santa Ana de Coro. Venezuela

Milena Pazmiño Freire; Cecilia Ivonne Narváez Zurita; Juan Carlos Erazo Álvarez

Figura 1. Esquema de gestión y control de inventarios para las bodegas y puntos de venta de las pequeña y medianas empresas productoras y comercializadoras de calzado

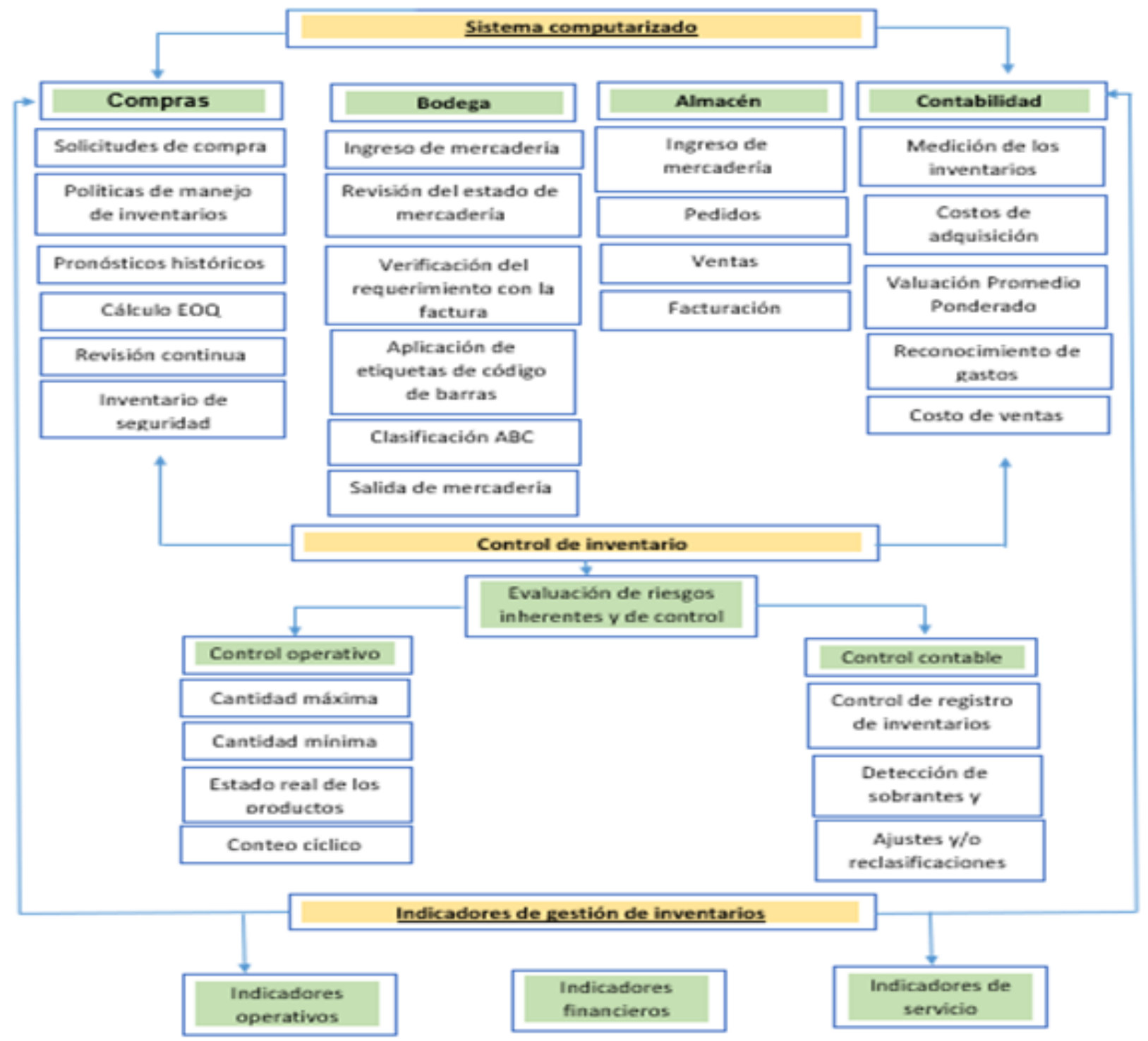




\section{CONCLUSIONES}

El inventario es uno de los elementos más importantes de las empresas manufactureras y comerciales que incide en su desempeño, por ende es importante mantener un inventario fiable, ya que esto permite tomar decisiones adecuadas, disminuir los excesos o faltantes de inventarios y ofrecer un servicio de calidad a los clientes.

En el caso específico del sector de calzado del cantón Cevallos de la provincia de Tungurahua el $84 \%$ de las pequeñas y medianas empresas tienen problemas en el manejo de sus inventarios ya sea por la deficiente organización de los productos en el almacén, por su clasificación de acuerdo al nivel de rotación, por la falta de registro oportuno de las transacciones, por la carencia de tecnologías que permitan simplificar las tareas de control de inventarios, o por el exceso de inventario, robos y desorden en las bodega. Por consiguiente con la finalidad de generar mayores beneficios para estas empresas se considera necesaria la adopción de mejores prácticas en el manejo de inventarios.

La investigación realizada a las pequeñas y medianas empresas productoras de calzado, localizadas en el catón Cevallos, muestra que las mismas no poseen mecanismos adecuados para la gestión y control de los inventarios, por lo que se dificulta la organización y clasificación del stock en los almacenes, así como la determinación razonable de los estados financieros.

El esquema propuesto para la gestión y control de inventarios considera varias aristas entre otras: la filosofía bajo la cual las pequeñas y medinas empresas productoras y comercializadoras de calzado van a dar respuesta a la cantidad a ordenar y el momento en que se debe realizar el requerimiento de adquisición en base al comportamiento de la demanda y las estrategias adoptadas; la incorporación de etiquetas de códigos de barras con la finalidad de identificar rápidamente los artículos, consultar características asociadas con los mismos y ejecutar inventarios de forma más rápida; la clasificación $A B C$ de los inventarios para segmentarlos según su nivel de importancia; la facturación electrónica a través de comprobantes de venta que garantizaran su autenticidad, origen 
e integridad del contenido; el tratamiento contable de los inventarios bajo las Normas Internacionales de Información Financiera; el control de inventarios a nivel operativo y contable, previo la determinación de los riesgos inherentes y de control. Finalmente incluye la incorporación de indicadores de gestión de inventarios para evaluar el desempeño de distintas variables y así disponer de información que permita tomar decisiones para controlar eficientemente el comportamiento de los inventarios.

\section{REFERENCIAS CONSULTADAS}

1. Arbós, L. (2012). Logística gestión de la cadena de suministros. Madrid, España: Díaz de Santos.

2. Arraiz, A. (2018). Framerwork de desarrollo de Proyectos Sociotecnológicos basado en la notación de Metamodelos de procesos de Ingeniería de Software (spem 2.0). Revista Arbitrada Interdisciplinaria Koinonía, 3(6), 79-95. Recuperado de

https://fundacionkoinonia.com.ve/ojs/index.php/revistakoinonia/article/view/141/1 21

3. Buston, C., y Chacón, G. (2007). Modelos deterministicos de inventarios para demanda independiente. Venezuela: Contaduría y administración.

4. Carmichael, D., Graham, L., y Whittington, R. (2007). Accountants' handbook financial accounting and general topics (11th ed.).

5. Cardona, J., Orejuela, J., y Rojas, C. (2018). Gestión de inventario y almacenamiento de materias primas en el sector de alimentos concentrados. EIA, 15(30).

6. Castro, C., Vélez, M., y Castro, J. (2011). Clasificación ABC Multicriterio: Tipos de Criterios y Efectos en la Asignación de Pesos. ITECKNE, 8(2), 163-170.

7. Cruz, A. (2017). Gestión de inventarios. Málaga: IC Editorial.

8. Chirinos, A. (2016). La innovación como factor clave de éxito en la gerencia de las empresas de producción social venezolana. CIENCIAMATRIA, 2(2), 97-106. https://doi.org/10.35381/cm.v2i2.55 
9. Durán, Y. (2012). Administración del inventario: elemento clave para la optimización de las utilidades en la empresa. Visión Gerencial, 55-78. Retrieved from https://www.redalyc.org/pdf/4655/465545892008.pdf

10. Ehrhardt, M., \& Eugene, B. (2007). Finanzas Corporativas. México: Thompson. Retrieved from https://www.academia.edu/22486512/Finanzas_Corporativas_2edi_Ehrhardt_FL_ redacted

11. Gaither, N., \& Frazier, G. (2012). Administración de producción y Operaciones (Octava Edición ed.). Madrid: Ediciones Paraninfo. Retrieved from https://apuntesutnpilar.files.wordpress.com/2012/09/administracion_de_producci on_y_operaciones.pdf

12. González, L. (2019). La herramienta que necesita su almacén. Retrieved from https://revistadelogistica.com/tecnologia/la-herramienta-que-necesita-sualmacen/

13. International Accounting Standard Board (IASB) (2015) IFRS. Normas Internacionales de Información Financiera (NIIF). Londres: IASCF Publications Department. - (2015). Marco Conceptual para la Información Financiera.

14. Juca, C., Narváez, C., y Erazo, J. (2019). Modelo de gestión y control de inventarios para la determinación de los niveles óptimos en la cadena de suministros de la empresa Modesto Casajoana Cía Ltda. 593 Digital Publisher, 1939.

15. Martínez, R. (2006). Gestión de inventarios y compras. MBA Ediciones.

16. Parada, O. (2009). Un enfoque multicriterio para la toma de decisiones en la gestión de inventarios. Cuadernos de Administración, 32(38). Retrieved 1010 , 2019

17. Peña , O., \& Silva, R. (2016). Factores incidentes sobre la gestión de sistemas de inventario en organizaciones venezolanas. Telos, 187-207.

18. Rodríguez, N., \& Gutiérrez, L. (2016). Análisis ABC en la gestión de inventarios : aplicación de la metodología en un caso específico. Grupo Ingenio Colombiano.

19. Sánchez, Á. (2017, Julio 29). Acerca de nosotros: aboutespanol.com. Retrieved from aboutespanol.com Web site: https://www.aboutespanol.com/que-es-unescaner-de-pc-841059 


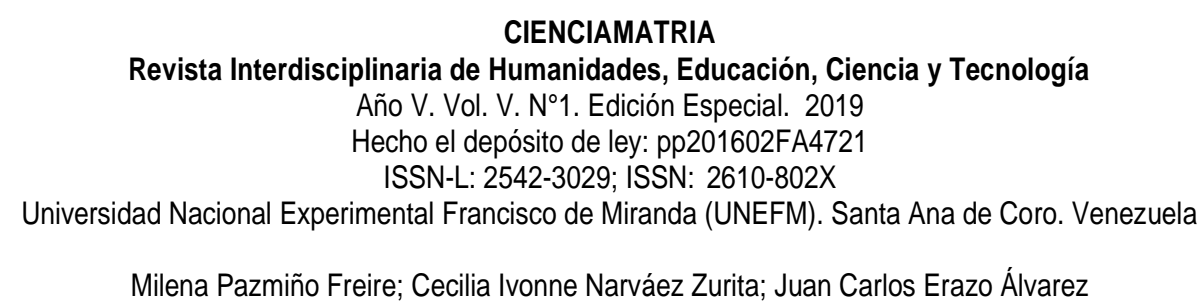

20. Schwarz, M. (2018). Sincronización de inventarios inteligentes en las cadenas de suministro . Perú: Universidad de Lima.

21. Sierra, J., Guzmán, M., \& García, F. (2017). Administración de almacenes y control de inventarios. Servicios Académicos Internacionales.

22. Toro, L., y Bastidas, V. (2011). Metodología para el control y la gestión de inventarios en una empresa minorista de electrodomésticos. Scientia et Technica Año XVI(49), 85-91.

23. Velázquez, L. (2012). Elaboración de una cédula como instrumento de gestión de Inventario . México D.F., Universidad Nacional Autónoma de México .

24. Veloz, C., \& Parada, O. (2017). Métodos para mejorar la eficiencia y la toma de decisiones en la gestión de inventarios. Ciencia UNEMI, 10(22), 29-38.

25. Vidal, C. (2010). Fundamentos de control y gestión de inventarios. Santiago de Cali, Colombia: Programa Editorial Universidad del Valle.

26. Weygandt, J., Kimmel P., y Kieso, D. (2009) Accounting principles (9th ed.). New York: Wiley

27.Zapata, J. (2014). Fundamentos de la gestion de inventarios. Medellin: Centro Editorial Esumer.

\section{REFERENCES CONSULTED}

1. Arbós, L. (2012). Logistics supply chain management. Madrid, Spain: Díaz de Santos.

2. Arraiz, A. (2018). Framerwork of development of Sociotechnological Projects based on the notation of Metamodels of Software Engineering processes (spem 2.0). Interdisciplinary Arbitrated Review Koinonía, 3 (6), 79-95. Recovered from https://fundacionkoinonia.com.ve/ojs/index.php/revistakoinonia/article/view/141/1 21

3. Buston, C., and Chacón, G. (2007). Deterministic inventory models for independent demand. Venezuela: Accounting and administration. 
4. Carmichael, D., Graham, L., and Whittington, R. (2007). Accountants 'handbook financial accounting and general topics (11th ed.).

5. Cardona, J., Orejuela, J., and Rojas, C. (2018). Inventory management and storage of raw materials in the concentrated food sector. EIA, 15 (30).

6. Castro, C., Vélez, M., and Castro, J. (2011). ABC Multicriteria Classification: Types of Criteria and Effects on Weight Assignment. ITECKNE, 8 (2), 163-170.

7. Cruz, A. (2017). Inventory management. Malaga: IC Editorial.

8. Chirinos, A. (2016). Innovation as a key success factor in the management of Venezuelan social production companies. SCIENCE, 2 (2), 97-106. https://doi.org/10.35381/cm.v2i2.55

9. Durán, Y. (2012). Inventory management: key element for the optimization of profits in the company. Management Vision, 55-78. Retrieved from https://www.redalyc.org/pdf/4655/465545892008.pdf

10.Ehrhardt, M., \& Eugene, B. (2007). Corporate Finance Mexico: Thompson. Retrieved from https://www.academia.edu/22486512/Finanzas_Corporativos_2edi_Ehrhardt_FL_ redacted

11. Gaither, N., \& Frazier, G. (2012). Production and Operations Administration (Eighth Edition ed.). Madrid: Paraninfo editions. Retrieved from https://apuntesutnpilar.files.wordpress.com/2012/09/administracion_de_producci on_y_operaciones.pdf

12. González, L. (2019). The tool your store needs. Retrieved from https://revistadelogistica.com/tecnologia/la-her tool-que-necesita-su-almacen/

13. International Accounting Standard Board (IASB) (2015) IFRS. International Financial Reporting Standards (IFRS). London: IASCF Publications Department. (2015). Conceptual Framework for Financial Information.

14. Juca, C., Narváez, C., and Erazo, J. (2019). Inventory management and control model for the determination of optimal levels in the supply chain of the company Modesto Casajoana Cía Ltda. 593 Digital Publisher, 19-39.

15. Martínez, R. (2006). Inventory and purchasing management. MBA Editions. 
16. Stop, O. (2009). A multi-criteria approach to decision making in inventory management. Administration Notebooks, 32 (38). Retrieved 10 10, 2019

17.Peña, O., \& Silva, R. (2016). Incident factors on the management of inventory systems in Venezuelan organizations. Telos, 187-207.

18. Rodríguez, N., \& Gutiérrez, L. (2016). ABC analysis in inventory management: application of the methodology in a specific case. Colombian Ingenio Group.

19. Sánchez, Á. (2017, July 29). About us: aboutespanol.com. Retrieved from aboutespanol.com Website: https://www.aboutespanol.com/que-es-un-escanerde-pc-841059

20. Schwarz, M. (2018). Synchronization of smart inventories in supply chains. Peru: University of Lima.

21. Sierra, J., Guzmán, M., \& García, F. (2017). Warehouse management and inventory control. International Academic Services.

22. Toro, L., and Bastidas, V. (2011). Methodology for inventory control and management in a home appliance retail company. Scientia et Technica Year XVI (49), 85-91.

23. Velázquez, L. (2012). Preparation of a certificate as an inventory management instrument. Mexico D.F., National Autonomous University of Mexico.

24. Veloz, C., \& Parada, O. (2017). Methods to improve efficiency and decision making in inventory management. UNEMI Science, 10 (22), 29-38.

25. Vidal, C. (2010). Fundamentals of inventory control and management. Santiago de Cali, Colombia: Editorial Program Universidad del Valle.

26. Weygandt, J., Kimmel P., and Kieso, D. (2009) Accounting principles (9th ed.). New York: Wiley

27.Zapata, J. (2014). Fundamentals of inventory management. Medellin: Esumer Editorial Center.

(C2019 por los autores. Este artículo es de acceso abierto y distribuido según los términos y condiciones de la licencia Creative Commons Atribución-NoComercial-Compartirlgual 4.0 Internacional (CC BY-NC-SA 4.0) (https://creativecommons.org/licenses/by-nc-sa/4.0/). 